\title{
Response Modes and Coordination in a Traffic Context, an Experimental Comparison of Chinese and German Participants.
}

Thorsten Chmura ${ }^{\mathrm{a} 1}$,

${ }^{a}$ Ludwig-Maximilians-Universität München, Seminar für Wirtschaftstheorie, Ludwigstraße 28, 80539

München

${ }^{\mathrm{b}}$ Laboratory of Experimental Economics, Adenauerallee 24-42, 53113 Bonn, Germany

Abstract: This paper reports results of laboratory experiments about traffic behaviour of participants with different cultural backgrounds. We conducted the minority game as an elementary traffic scenario in which human participants of a German and Chinese subject pool had to choose over 100 periods between a $\operatorname{road} A$ and a road $B$. In each period, the road which was chosen by the minority of players won, these participants got a payoff. The payoff in the majority group was 0 . An important observation is that the number of road changes of a participant is negatively correlated with her cumulative payoff. The Chinese participants reacted differently to the payoffs of preceding periods than the German participants.

Keywords: minority game, laboratory experiments, traffic behaviour, cross cultural experiments, comparison of coordination behavior

JEL classification: C88; C91; C92; C15; D83; R40; R41

\footnotetext{
${ }^{1}$ Thorsten Chmura: Phone: +49(0) 89/2180-3907 - Fax: +49(0)89/2180-3510 - Email: thorsten.chmura@1rz.uni-muenchen.de
} 


\section{Introduction}

This paper reports about laboratory experiments concerning traffic behaviour of participants with different cultural backgrounds. We used a classifier system for behavioural types, which was introduced by Selten et al. (2007). It can be shown that different cultural backgrounds may have an influence on the cognitive decision process in binary choice situations; we used a route choice scenario. Two subject pools with 54 participants each were analysed:

1. German students at the University of Bonn (Germany).

2. Chinese students at the Shanghai Jiao Tong University and Nankai University (China).

Obviously the traffic situation in Chinas densely populated cities differs from the German and most Central European areas. The traffic in China's cities is much more heterogeneous. Especially in Shanghai are more bikes, motorcycles, pedestrians, cars and busses on the road at the same time. In Germany as in most other countries of the European Union there are often extra lanes for busses, taxies and bikes. Our approach is not comparing the traffic situations inherently, but it could give a better understanding whether traffic participants in China act more anticipatory in view of the more complex situation on the roads, than the German traffic participants. It seems necessary to react in a different way in China.

During the last two decades cross-cultural comparisons and differences had been studied. Empirical data was used to gain a deeper inside of different countries. The investigation 
of the economic performance and the social capital in different countries and nations was the target in several studies. Gambetta (1988), Putnam (1993), and Fukuyama (1995, 2001) shown that the social capital affects the performance and the economic success of organizations and the economy. La Porta et al. (1997), and Fukuyama (1995, 2001) reported that social and civic norms of cooperation have an impact on institutions and aggregate economic activity.

Cross-cultural studies using an experimental setup have become an important field in economics research. The most common experimental designs are various specifications of the ultimatum game, the trust game, the dictator game and public good games example in Buchan (1997), Botelho (2000), Burns (2004), Carpenter (2004), Chuah (2005), Henrich (2000, 2001, 2004), Oosterbeek (2004) and Roth (1991). The authors report that social norms depend on the cultural background of participants. Camerer (2003) remarks that offers and counteroffers are a language with nuance and cultural variation in bargaining experiments.

A comparison between the Western and Asian cultures is discussed in Hofstede, (1980). The authors conclude that the relative dominance of individual versus collective interests is the explanation of the differences in the cultural behavior. Some Business studies use the Confucian value system and the Chinese socio-economic structure as an explanation for the cultural differences in negotiation and decision-making processes e.g. (Tung, 1989; Child and Marcoczy, 1994).

Important attributes of cross-cultural experimental studies (e.g. Anderson et al., 2000; 
Henrich, 2000; Henrich et al., 2001; Buchan et al., 2004a,b) is the sequential application of an identical experimental setup within selected areas while controlling for stakes, language, experimenter effects and demographic background variables (Roth et al., 1991, Camerer and Kagel, 1995; Walkowitz et al., 2004; Dakkak et al., 2006).

In our study we designed a simple minority game to explore different response modes of a Chinese and a German group of participants. The two response modes (chapter 3.2) will explain payoff and coordination differences between the cultural groups.

Characteristic for a traffic situation worldwide is that many subjects have to interact without a negotiation procedure. Since there is an inherent lack of communication, optimal coordination is rather unlikely. The only way to increase individual benefit, what means to decrease individual travel time, is to adapt individual decisions to the behavior of the other participants; which could be observed in the past. To model such a situation we used a simple Minority Game.

The Minority Game is an example of a $n$-person game with no strict pure equilibria and can be applied on different situations including their specific social and economic contexts. The Minority Game, which is also called the El Farol Bar Problem and was introduced by Arthur (1994) and theoretically analysed in detail by Challet \& Zhang, (1997, 1998). There is already some literature about experimental studies of the game. Helbing et al. (2005), Renault et al. (2005), Chmura \& Pitz (2006), Bottazzi \& Devetag (2007) and Kets \& Voornfeld (2007). 
The rules of the Minority Game can be described in a short way: a number of agents $n$ have to choose during several periods whether to enter a given room A or a room B. Those agents who choose the less crowded room win whereas the others lose. Our aim is to present Minority Game experiments with a large number of periods and with sufficiently many independent observations for meaningful applications of nonparametric significance tests.

Market entry games (Rapoport et al 2002, Erev and Rapoport 1998) are another kind of games found in experimental literature, which can be compared in some aspects with the Minority Game. In these types of games players usually have the choice either to enter a market or to stay out of it. The payoff for entering the market is a decreasing function of the number of entrants. The payoff for staying out is a constant opportunity cost. One may say that the route choice game is similar to a market entry game with two markets instead of one. However, the players do not have the choice to stay out of both markets.

A number of experiments on route choice behaviour could be found in the literature (e.g. Bonsall 1992, Mahmassani \& Liu 1999, Selten et al. 2007, Chmura \& Pitz 2006). Here, we focus on the route choice behaviour in a generic two route scenario, which has already been investigated in the scientific literature (e.g. Iida et al. 1992). In Helbing et al (2002) volatile dynamics of decisions independent of an optimal payoff distribution were observed in route choice experiments. It could be shown that specific guidance strategies are able to increase the performance of all users by reducing overreaction and stabilizing 
the decision dynamics. In De Martino (2004) a model for analysing the emergent collective behaviour of drivers in a city was discussed. The results proved that in absence of information noise, inductive drivers turn out to behave in a more effective way than random drivers during periods of low car density, while high car densities produce the opposite effect.

In this paper, special emphasis shall be laid on a comparison of the participants' reactions to the immediately preceding payoffs. The results showed that differences in behaviour are observed between the culturally divergent groups.

\section{Experimental setup}

The experiments were conducted during September and November 2006. The German sessions were run at the BonnEconLab at the University of Bonn, Germany. The first three Chinese sessions are located at the Reinhard Selten Lab at Nankai University of Tianjin, China and the Chinese sessions 4, 5, 6 are located at the Vernon Smith Experimental Lab of the Shanghai Jiao Tong University. At the 3 universities students from several departments participated.

Experiments were run by local helpers comprehensively instructed and supported by the authors, who stayed in the background. We are aware that this might result in an experimenter effect. We decided to choose this procedure to avoid self-presentation and face-saving effects Bond \& Hwang (1986) of inexperienced subjects resulting from the

presence of people from foreign countries. Since we are interested in the pure 
presentation effect this procedure seems to be justified. Instructions were written in neutral language.

An anonymous referee asked whether the Germans are more likely to own a license to drive a car than the Chinese students. We did not control this question in our experiment. It is more likely that more German participants hold a drivers license for a car. We agree that this question would have been interesting, but all of he subjects are part of their local traffic network, whether they use a car, taxi, bike, e-bike or even walk. We believe that every traffic participant can easily understand the setup of this treatment since no one likes to be in a traffic jam. We are thankful for the comment of the anonymous referee, because in further investigations we will control this question.

To avoid translation errors regarding the task and the cadence instructions were translated by natural speakers from German into the corresponding language and afterwards translated back into German applying the back-translation method (Brislin, 1970).

For this survey, the Minority Game was transferred to a route choice context Chmura \& Pitz (2006). In these experiments, subjects were told that in each of the periods 0 to 100 , they had to make a choice between a $\operatorname{road} A$ and a $\operatorname{road} B$ for travelling from $\mathrm{X}$ to $\mathrm{Y}$. Six sessions were run with German and six sessions with Chinese participants. The number of subjects in each session was 9 . They were told that the travel times $t_{A}$ and $t_{B}$ on $\operatorname{road} A$ and $B$ depended on the numbers $n_{A}$ and $n_{B}$ of participants choosing $A$ and $B$ respectively: 


$$
t_{A}=1, t_{B}=0 \Leftrightarrow n_{A}<n_{B} \text { and } t_{B}=1, t_{A}=0 \Leftrightarrow n_{A}>n_{B} .
$$

The period payoff was $t_{A}$ if $A$ was chosen and $t_{B}$ if $B$ was chosen. The total payoff of a subject was the sum of all period payoffs in Taler (experimental currency unit) converted proportionally to monetary payoffs in Euro respectively RMB. The conversion rate was 1 Taler $=0.4 €$ in Germany and 1 Taler $=2 \mathrm{RMB}$ in China. The difference of the conversion rate can be explained by the laboratory standard payoff in each country. The experimental data were obtained in 12 sessions with 9 subjects each and 108 altogether. The computer program was based on the toolbox RatImage developed by Abbink \& Sadrieh (1995).

After each of the 100 periods subjects received information about the preceding period, the period payoff in Taler, the cumulative payoff and the number of the past period. We did not provide any further information.

\section{Experimental results}

In this section, we explain the main statistical findings while later in the subchapters we will explain the results in view of the response modes and the cumulative payoff.

\subsection{Descriptive statistics for the Chinese and the German treatment}

The basic statistical findings are shown in table 1 . Figure 1 shows the number of participants on road $A$ as a function of time for a typical observation of the Chinese participants and the German group. The mean number of players on road $A$ is 4.5 in the 
Chinese group and 4.49 in German group. This was the expected outcome since the experimental setup does not suggest a preference for one road. The Minority Game with 9 players has $2\left(\begin{array}{l}9 \\ 4\end{array}\right)=252$ (non strict) Nash equilibria in pure strategies.

The lack of strict pure strategy equilibria poses a coordination problem that may be one of the reasons for non-convergence and the persistence of fluctuations in both treatments. The mean number of players for the Chinese and the German observations are shown in figure 2. There is no significant difference between the German and the Chinese treatment for the mean numbers of players choosing the road A.

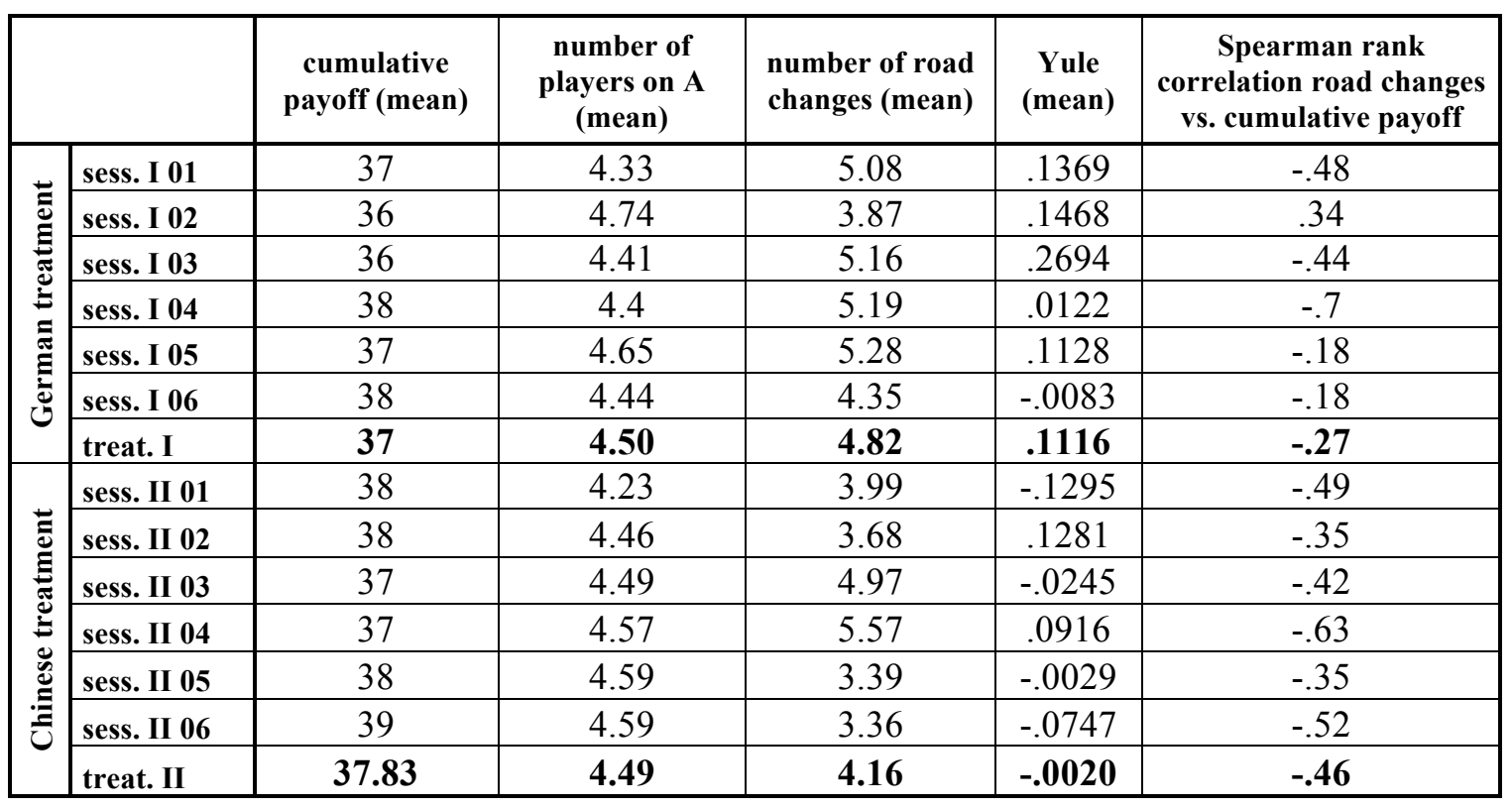

Table 1: Statistical data of the experiments.

It seems that there is no outstandingly advisable strategy for the participants to enhance their payoffs because due to the symmetry of the game, each road has the same properties. However, one can see in the next section that in some cases, certain types of reactions to former payoffs are more successful than others. 

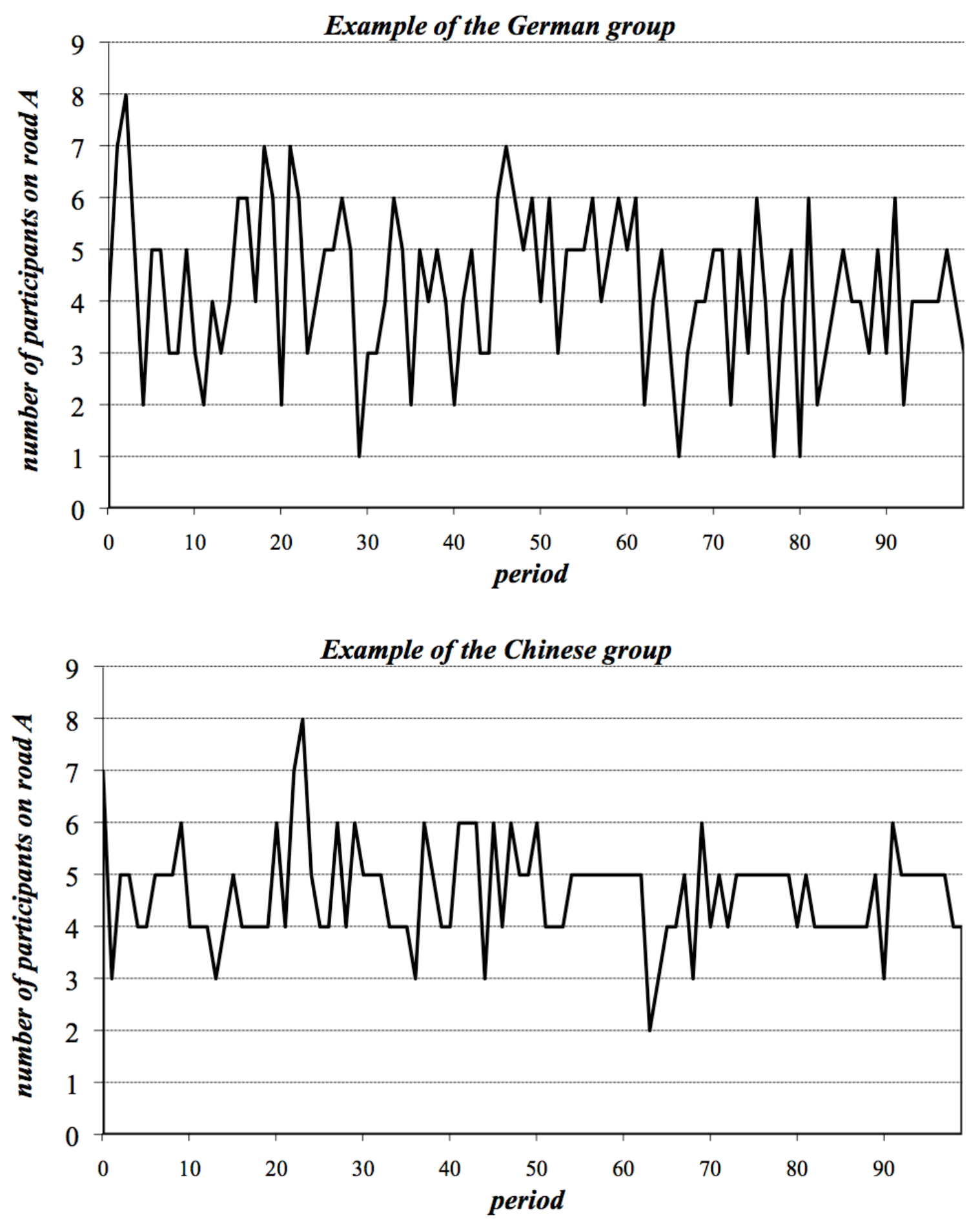

Figure 1: Number of participants on $A$ : a typical session of the German and the Chinese group. 
Table 1 also shows the mean number of road changes, the mean Yule-coefficient and cumulative payoff as well as the spearmen rank correlation coefficient for the number of road changes versus the cumulative payoff. All these values for the German treatment are significantly different from the Chinese treatment. We will try to explain this in section 3.2 and 3.3

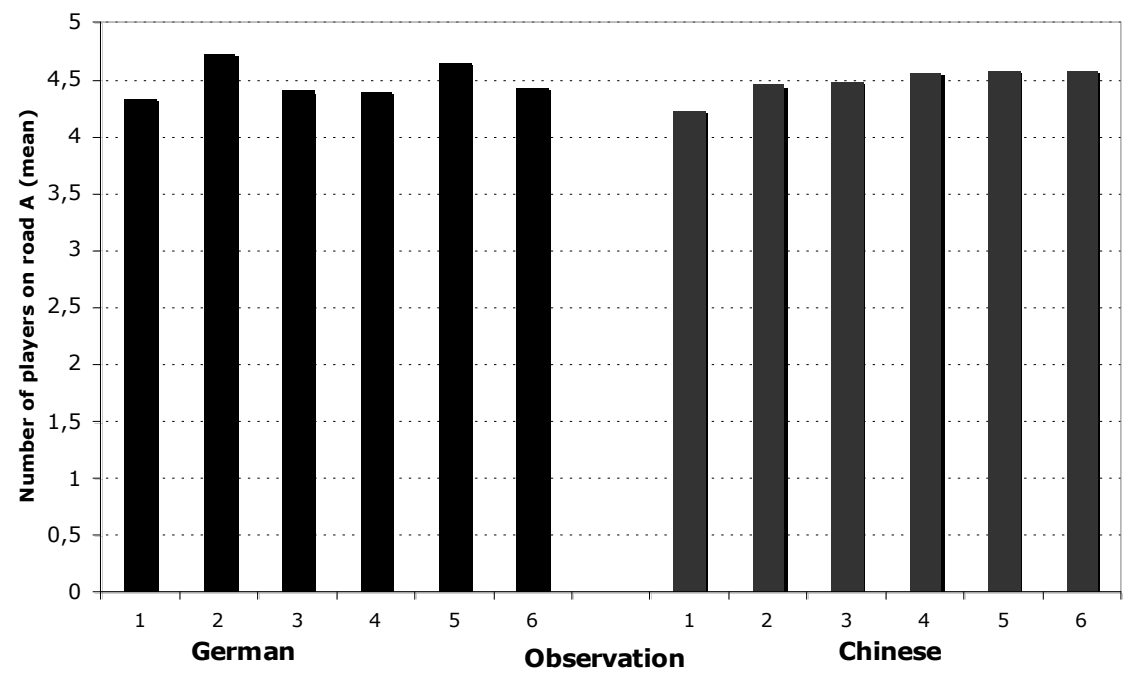

Figure 2: Number of participants on road A for the German and the Chinese treatment.

\subsection{A classifier system of response modes}

We used a classifier system for behavioural types introduced by SELTEN ET AL. (2007) to describe reactions of former payoffs. The classifier system can be described as follows: A participant who had a payoff 0 (1) on the road chosen may change the road (stay on the same road) in the next period in order to travel on a less crowded route. We call this the direct response mode. The direct response mode is the prevailing one but there is also a contrarian response mode. The contrarian participant expects that a payoff $1(0)$ will 
attract (deter) many others and that therefore the road chosen will be crowded (free) in the next period.

For each subject, let $c_{-}\left(c_{+}\right)$be the number of times in which a subject changes the roads when the payoff in the period before was $p=0(p=1)$. And for each subject let $\mathrm{s},\left(s_{+}\right)$be the number of times in which a subject stays on the road when there was a payoff $p=0(p=1)$ in the period before.

\begin{tabular}{|c|c|c|}
\hline & change & stay \\
\hline $\mathbf{p}=\mathbf{0}$ & $c_{-}$ & $s_{-}$ \\
\hline $\mathbf{p}=\mathbf{1}$ & $c_{+}$ & $s_{+}$ \\
\hline
\end{tabular}

Table 2: $2 \times 2$ table for the computation of Yule-coefficients.

For each subject such a 2x2 table has been determined and a Yule-coefficient $Q$ has been computed as follows.

$$
Q=\frac{c_{-} \cdot s_{+}-c_{+} \cdot s_{-}}{c_{-} \cdot s_{+}+c_{+} \cdot s_{-}}
$$

The Yule coefficient has a range from -1 to +1 . Participants with a "high" Yulecoefficient near to $1(-1)$ tend to be direct (contrarian).

The classifier system used in this paper is able to show contrarian and direct responses. It does not classify individuals. A direct response of a participant is counted as a direct response. It is possible that the same participant is half of the experiment a direct responder and in the second half a contrarian responder. 


\subsection{Observed Response mode}

To classify behavioural types we used the Yule-coefficient we described this already in section 3.2. The mean Yule-coefficients are significantly higher in the German treatment (see figure 4). The null-hypothesis for both treatments is rejected by a Wilcoxon-MannWhitney-Test on the significance level of 5\% (one-sided). That means that there are less contrarian response modes in the German treatment.

The reason for the smaller Yule-coefficients in the Chinese treatment lies in the fact that contrarian reactions to former payoffs occur more frequently in this group. One can see in table 1 that the number of road changes per round in the German treatment is significantly higher than in the Chinese treatment. The null-hypothesis for both treatments is rejected by a Wilcoxon-Mann-Whitney-Test on the significance level of $1 \%$ (one-sided). Since the players' mean payoff (for all the experiments) is 37.41 and since therefore a player receives more „bad“ than „good“ payoffs on average, the decline in road changes in the treatment of Chinese participants is another indicator for an increase of contrarian behavioural types. The number or road changes for both treatments is graphically shown in figure 3. 


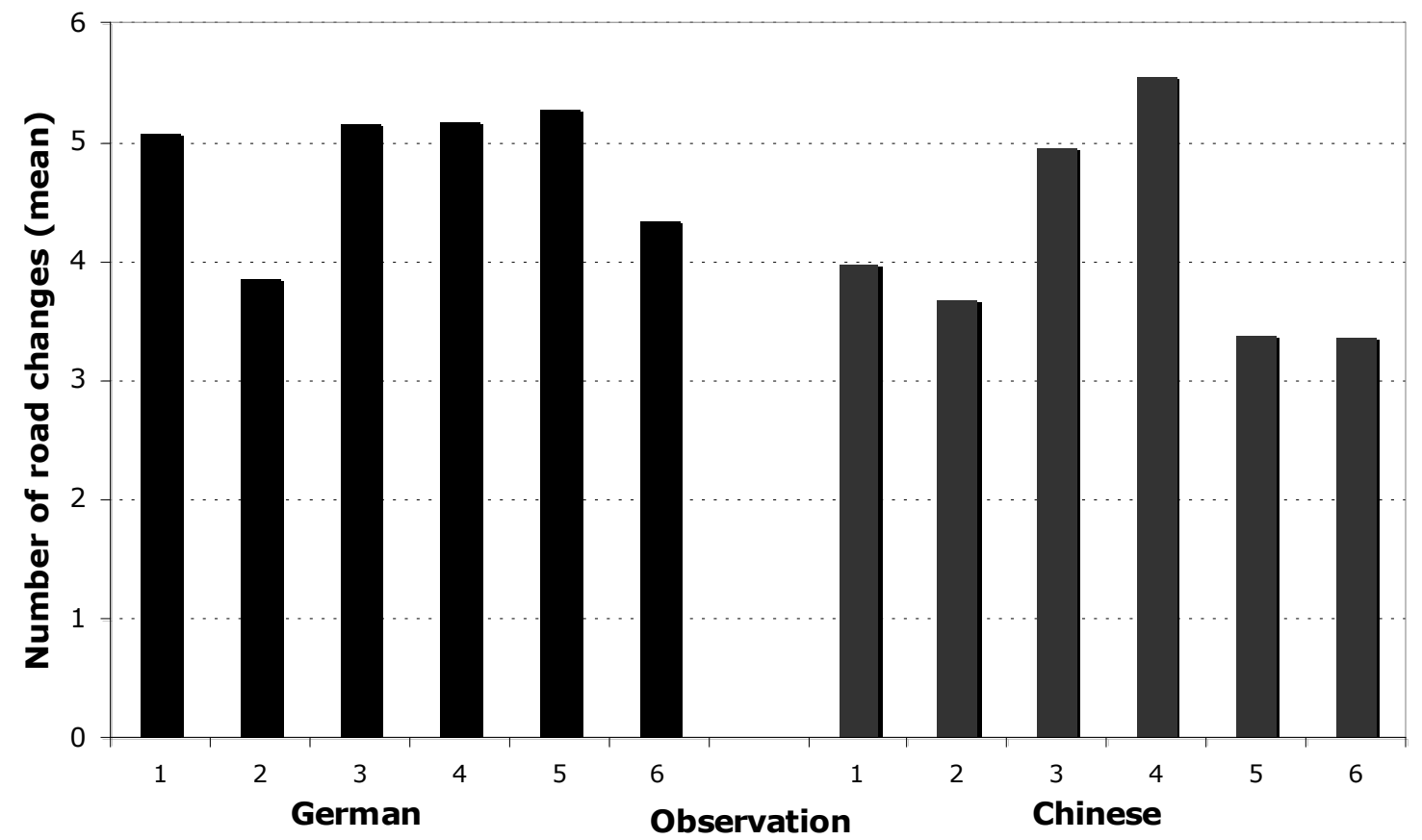

Figure 3: Mean number of road changes for the German and the Chinese treatment.

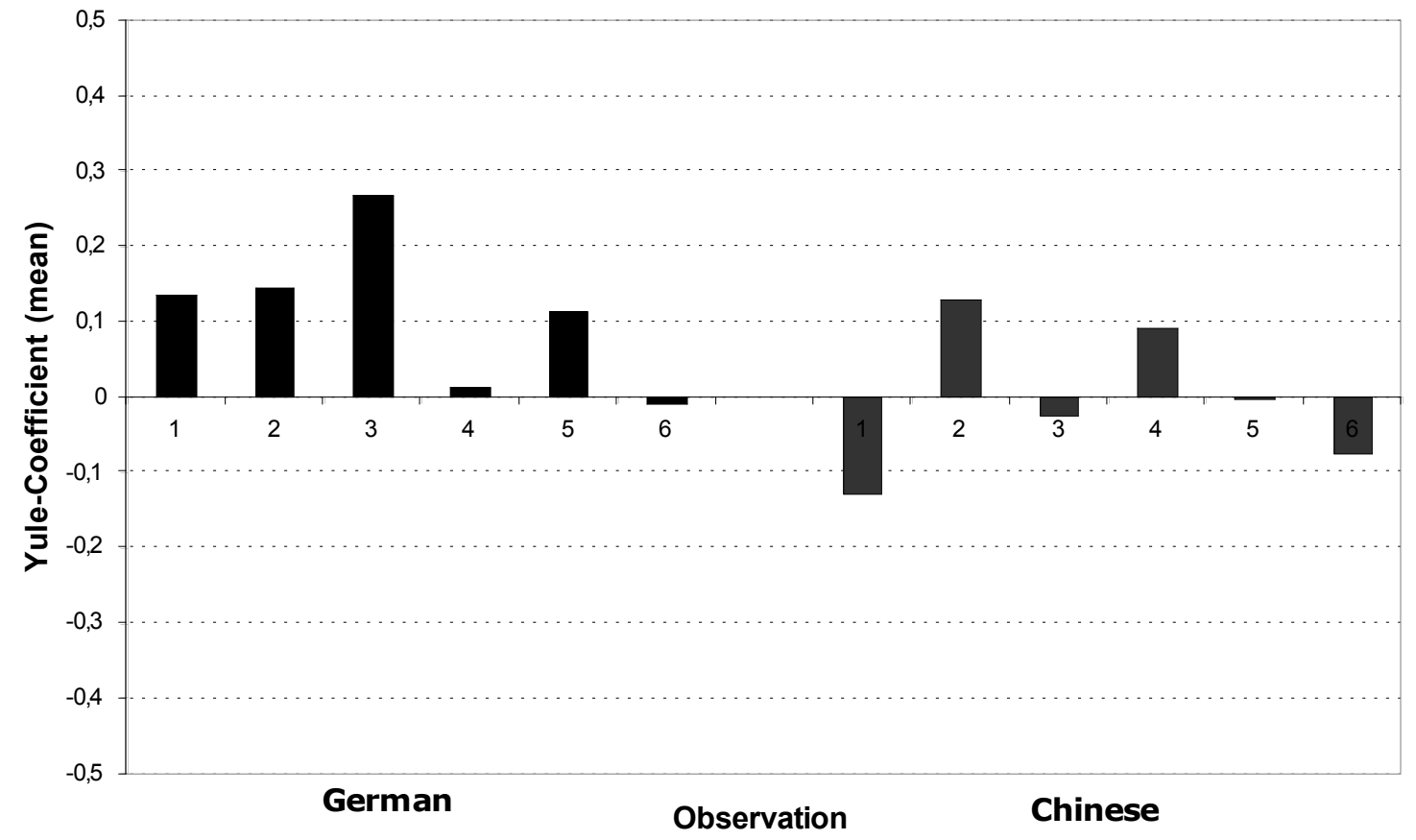

Figure 4: Yule-Coefficient for the German and the Chinese treatment. 


\subsection{Cumulative Payoff}

In Chmura \& Pitz (2006), it was already pointed out that a negative correlation exists between the cumulative payoff and the frequency of road changes of a player. Figure 5 shows the mean cumulative payoff for the German and the Chinese treatment. As shown in table 1, the Spearman rank correlation coefficient is negative for all Chinese sessions and 5 German sessions. This also is shown in figure 6. Since the contrarian response mode could be observed more frequent in the Chinese treatment and thus, the number of "good" payoffs was on average higher than of the "bad" payoffs, it could be expected that the Chinese players would on average receive better results than the group with German participants. Indeed, table 1 shows that the mean payoff per session is higher in Chinese observations than in the German observations. The related null-hypothesis was rejected by a Wilcoxon-Mann-Withney-test on the significance level of 5\% (one-sided). In the case of the Minority Game, the contrarian response mode of the Chinese participants is the more promising strategy. 


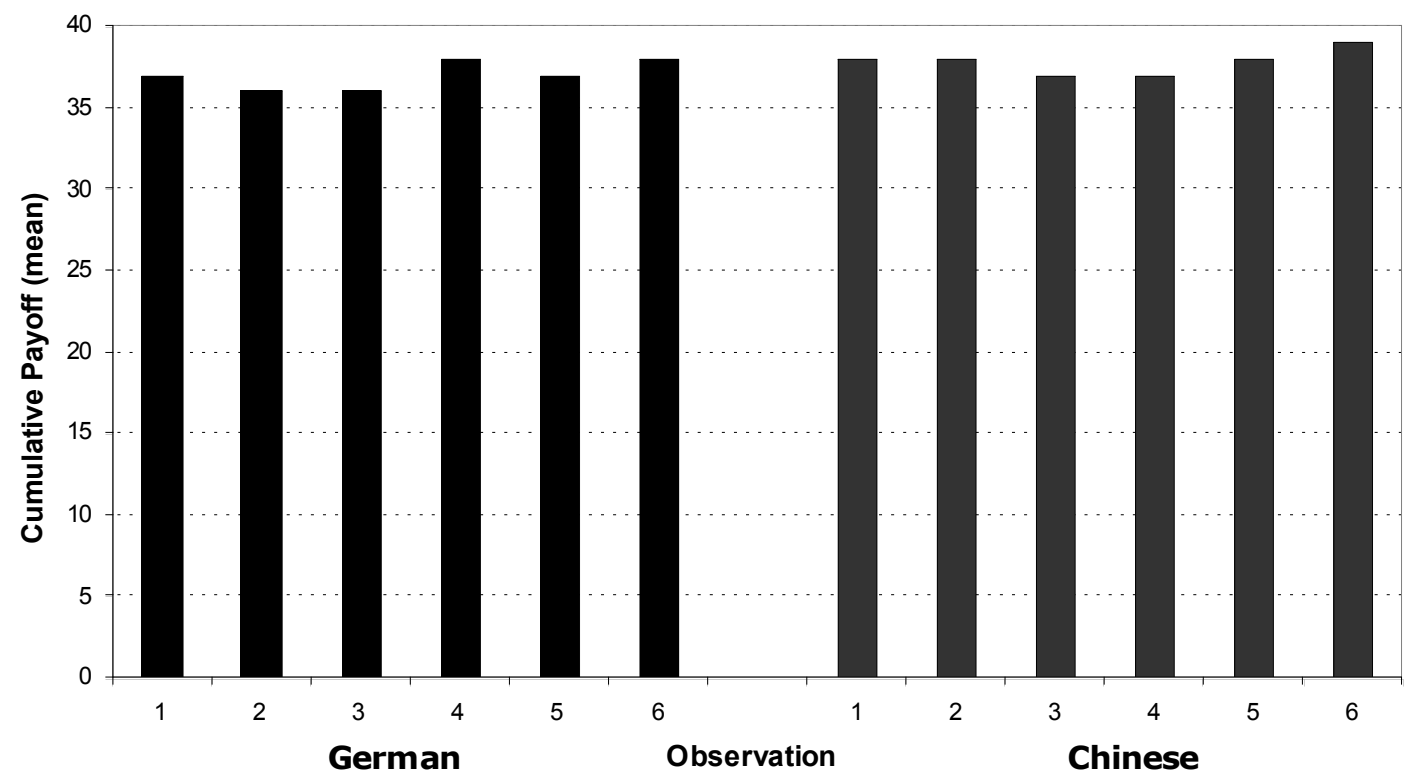

Figure 5: Mean cumulative payoff for the German and the Chinese treatment.

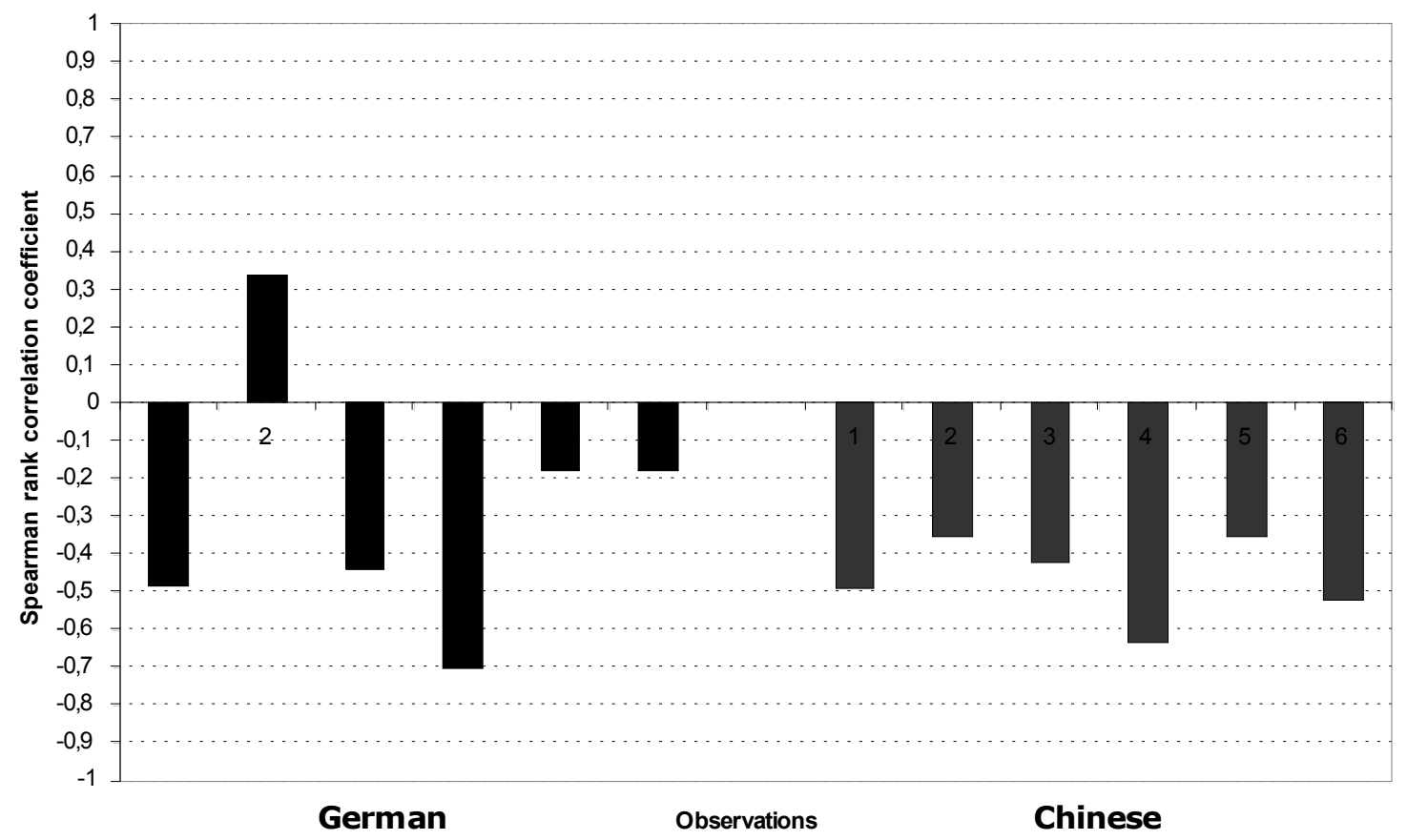

Figure 6: Spearmen rank correlation coefficient for the cumulative payoff vs. the number of road changes. 


\section{Conclusion}

In this paper we discussed an elementary traffic scenario, modelled as a minority game with subjects of different cultural backgrounds. We found two response modes using the Yule-coefficient. The first response mode is a direct response and the second a contrarian response to the received payoff in the last period. The reactions of participants of the two investigated groups were significantly different. The German subjects reacted in a more direct way than the Chinese, i. e. by the above definition of direct, that they chose the same road after good payoffs and changed after bad payoffs. Due to the different behaviour and the structure of the minority game the average payoff of the German subjects in this game was lower than the average payoff of the Chinese. The less direct reactions of the Chinese participants may be caused by their different experience in their daily traffic situation. In a crowded inhomogeneous traffic situation a contrarian reaction, which anticipates, the possible reactions of the other participants more severely than the direct response mode, seems to be reasonable. To our knowledge the comparison of an Asian (Chinese) group to a Western (German) group using a response mode as a classification scheme was not conducted before. Our results show that groups with a different cultural or local background react different to given information, here whether they have a payoff or not. We used the classification scheme in traffic context in a minority game. The reaction of traffic participants to information (radio, gps or the internet) is important to develop better precast systems for traffic routes. The game we used was simple and a very limited model compared to the real life traffic. One can assume that the different response modes and the different reactions to the given information (here just the payoff) may lead to a stronger reaction in the daily route choice 
behaviour. The finding that the Chinese group responds in a more contrarian way than the German group is an important insight and needs further research, whether this holds true for other games and situations as well.

\section{References}

Abbink, K., and A. Sadrieh (1995). RatImage—Research Assistance Toolbox for Computer-Aided Human Behavior Experiments. University of Bonn SFB Discussion Paper B-325.

Anderson, L. R., Rodgers, Y. V., and Rodriguez, R. R. (2000). Cultural differences in attitudes toward bargaining. Econ. Letters 69, 45-54.

ArThUR, W.B. (1991). Designing Economic Agents That Act Like Human Agents: A Behavioral Approach to Bounded Rationality, American Economic Review, vol. 81(2), pages $353-59$.

Bond, M. H. and K. Hwang (1986). The psychology of the Chinese people, in M. H. Bond (Ed.): The social psychology of the Chinese people, Oxford University Press, Hong Kong, pp. 213-266. 
Bonsall, P. (1992). The influence of route guidance advice on route choice in urban networks, Transportation 19, 1-23.

Botelho, A., Hirsch, M. A., Ruström, E. E. (2000). Culture, nationality and demographics in ultimatum games. December 2000.

Brislin, R. W. (1970). Back-translation for cross-cultural research, Journal of Crosscultural Psychology, No. 1, 185-216.

Buchan, N. R., E., J., Croson, R. T. A. (1997). Culture, power and legitimacy: Contrasting influences on fairness beliefs and negotiation behavior in Japan and the United States, University of Wisconsin Department of Marketing Working Paper.

Bottazzi, G. and G. Devetag (2007). Competition and coordination in experimental minority games. Journal of Evolutionary Economics 17, $241-275$.

Burns, J., (2004). Trust and reciprocity in Post-Apartheid SouthAfrica, working Paper.

Buchan, N., Croson, N. T. A., and Johnson, E. J. (2004a). When do fairness beliefs influence bargaining behavior: Experimental evidence from Japan and the United States. J. Cons. Res. 31, 181-190. 
Buchan, N., Croson, N. T. A., and Johnson, E. J. (2004b). The boundaries of trust: Own and other's actions in the US and China. J. Econ. Behav. Organ. 55(4), 485-504.

Carpenter, J. P., Daniere, A. G., Takahashi, L. M. (2004). Cooperation, trust, and social capital in Southeast Asian urban slums. Journal of Economic Behavior and Organization $55(4), 533-551$.

Challet, D., Zhang, Y.-C., (1997). Emergence of cooperation and organization in an evolutionary game. Physica A 246, 407-418.

Challet D., Zhang, Y.-C., (1998). On the minority game: Analytical and numerical studies. Physica A 256, 514-532.

Camerer, C., Kagel, H. K. (1995). The handbook of experimental economics. Princton University Press, New York.

Child, J. and L. Marcoczy (1994). Host Country Managerial Behaviour in Chinese and Hungar- ian Joint Ventures: Assessment and Competing Explanations. In: Boisot, M. (ed.): East- West Business Collaboration: The Challenge of Governance in Post-socialist Enterprises. Routledge, London and New York, 25-50.

Chmura, T., Pitz T. (2006). The Successful Stoics - An Astonishing Property of the Minority Game, Physica A 363, 477-480. 
Chuah S., Hoffmann R., Jones M, Geoffrey Williams G. (2005). An Economic Anatomy of Culture: Attitudes and Behaviour in Inter- and Intra-National Ultimatum Game Experiments, CeDEx Discussion Paper No. 2005-11.

Dakkak, I., Hennig-Schmidt, H., Selten, R., Walkowitz, G., and Winter, E. (2006). Actions and beliefs in a trilateral trust game involving Germans, Israelis, and Palestinians. Working Paper, University of Bonn.

De Martino A., Marsili M., Mulet R. (2004). Adaptive drivers in a model of urban traffic, Europhys Lett. 65. 283.

Erev, I., Rapoport, A., (1998). Coordination, "magic", and reinforcement learning in a market entry game. Games and Economic Behavior, 23, 146-175.

Fukuyama, F. (1995). Trust: The social virtues and the creation of prosperity. The Free Press, New York.

Fukuyama, F. (2001). Social capital, civil society and development. Third World Quarterly 22(1), 7-20.

Gambetta, D. (1988). Can we trust? In Gambetta, D. (Ed.), Trust. Basil Blackwell, New York, pp. 213-237. 
Helbing D., Schönhof M., Kern D., (2002). Volatile decision dynamics: experiments, stochastic description, intermittency control and traffic optimization, New J. Phys. 433.

Helbing, D., M. Schönhof, H.-U. Stark, and J. A. Holust (2005). How individuals learn to take turns: Emergence of alternating cooperation in a congestion game and the Prisoners Dilemma. Advances in Complex Systems 8, $87-116$.

Hofstede, G. (2001). Culture's consequences: Comparing values, behaviors, institutions and organizations across nations. Sage Publications, Thousand Oaks.

Iida, Y., T. Akiyama, and T. Uchida. (1992). Experimental analysis of dynamic route choice behaviour. Transp. Res. B 26, 17-32.

Henrich, J. (2000). Does culture matter in economic behavior? Ultimatum game bargaining among the Machiguenga. American Economic Review 90 (4), 973-979.

Henrich, J., Boyd, R., Bowles, S., Camerer, C., Fehr, E., Gintis, H. (EDS.), (2004). Foundations of Human Sociality: Economic Experiments and Ethnographic Evidence from fifteen Small-Scale Societies. Oxford University Press, Oxford, UK.

Henrich, J., Boyd, R., Bowles, S., Camerer, C., Gintis, H., McElreath, R., Fehr, E. (2001). In search of homo economicus: Experiments in 15 smallscale societies. American 
Economic Review 91 (2), 73-79.

Hofstede, G. (2001). Culture's consequences: Comparing values, behaviors, institutions and organizations across nations. Sage Publications, Thousand Oaks.

Kets, W., Voorneveld, M. (2007). Congestion, equilibrium and learning: The minority game, Working Paper.

La Porta, R., De Silanes, F. L., Shleifer, A., and Vishny, R. W. (1997). Trust in large organizations. Amer. Econ. Rev. 87(2), S. 333-338.

Mahmassani, H.S., Liu, Y. H. (1999). Dynamics of commuting decision behaviour under advanced traveller information systems. Transp. Res. C 7, 91-107.

Oosterbeek, H., Sloof, R., van de Kuilen, G., (2004). Cultural differences in ultimatum game experiments: evidence from a meta-analysis. Experimental Economics 7, 171-188.

Putnam, R. D. (1993). Making democracy work: Civic traditions in modern Italy. Princeton University Press, New York.

Renault, J., S. Scarlatti, and M. Scarsini (2005) A folk theorem for minority games. Games and Economic, Behavior 53, $208-230$. 
Roth, A. E., Prasnikar, V. Okuno-Fujiwara, M., Zamir, S., (1991). Bargaining and market behavior in Jerusalem, Ljubljana, Pittsburgh and Tokyo: An experimental study. American Economic Review 81, 1068-1095.

Selten, R. Schreckenberg, M. Chmura, S. Kube, T. Pitz, T. (2007). Commuters Route Choice Behaviour, Games and Economic Behaviour 58, 394-406.

Tung, R. (1989). A Longitudinal Study of United States-China Business Negotiations, China-Economic-Review; 1(1), 57-71.

Walkowitz, G., Oberhammer, C., and Hennig-Schmidt, H. (2004). Experimenting over a long distance - A method to facilitate intercultural experiments. Bonn Econ Discussion Paper No. 17/2004, University of Bonn. 


\title{
Appendix
}

\section{Appendix A: Leaflet to minority experiment}

\section{Leaflet to minority experiment}

\author{
Welcome to the experiment
}

Procedure:

Altogether 9 persons are participating in this experiment. The game situation is the same for every participant.

The experiment consists of $\mathbf{1 0 0}$ periods.

In each period you are travelling from a starting point $X$ to an arrival point $Y$. You can either choose road $\mathbf{A}$ or road $\mathbf{B}$ to get from $\mathbf{X}$ to $\mathbf{Y}$ (see drawing).

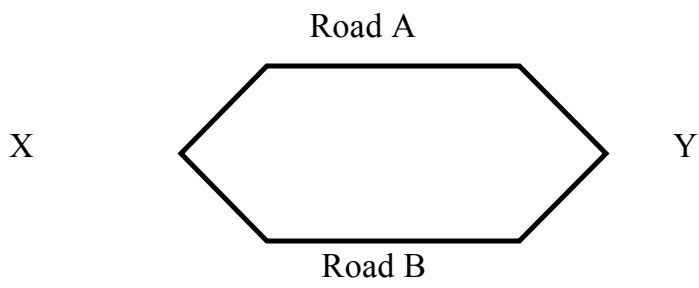

After your decision which road you choose, you will get a payoff if you are on the road, which the minority has chosen. In this game 9 players interact with each other. An example would be:

- $\quad 3$ participants choose road A and 6 participants choose road B, then each of the 3 participants on road A get the payoff of 1 Taler and the 6 participants on road B get the payoff 0 Taler.

5 participants choose road A and 4 participants choose road B, then each of the 4 participants on road B get the payoff 1 Taler and the 5 participants on road A get the payoff 0 Taler.

You can make a new route choice in every period.

The following information you will get after each period:

- Your route chosen in the preceding period.

- Your period payoffs in the preceding period in Talers.

- Your cumulated payoffs before the route choice in Talers.

- Number of the current period.

The exchange rate is $0,40 €(2 \mathrm{RMB}$ in the Chinese treatment) per Taler.

Thank you for participating! 


\section{Appendix B: Screenshot Of The Program}

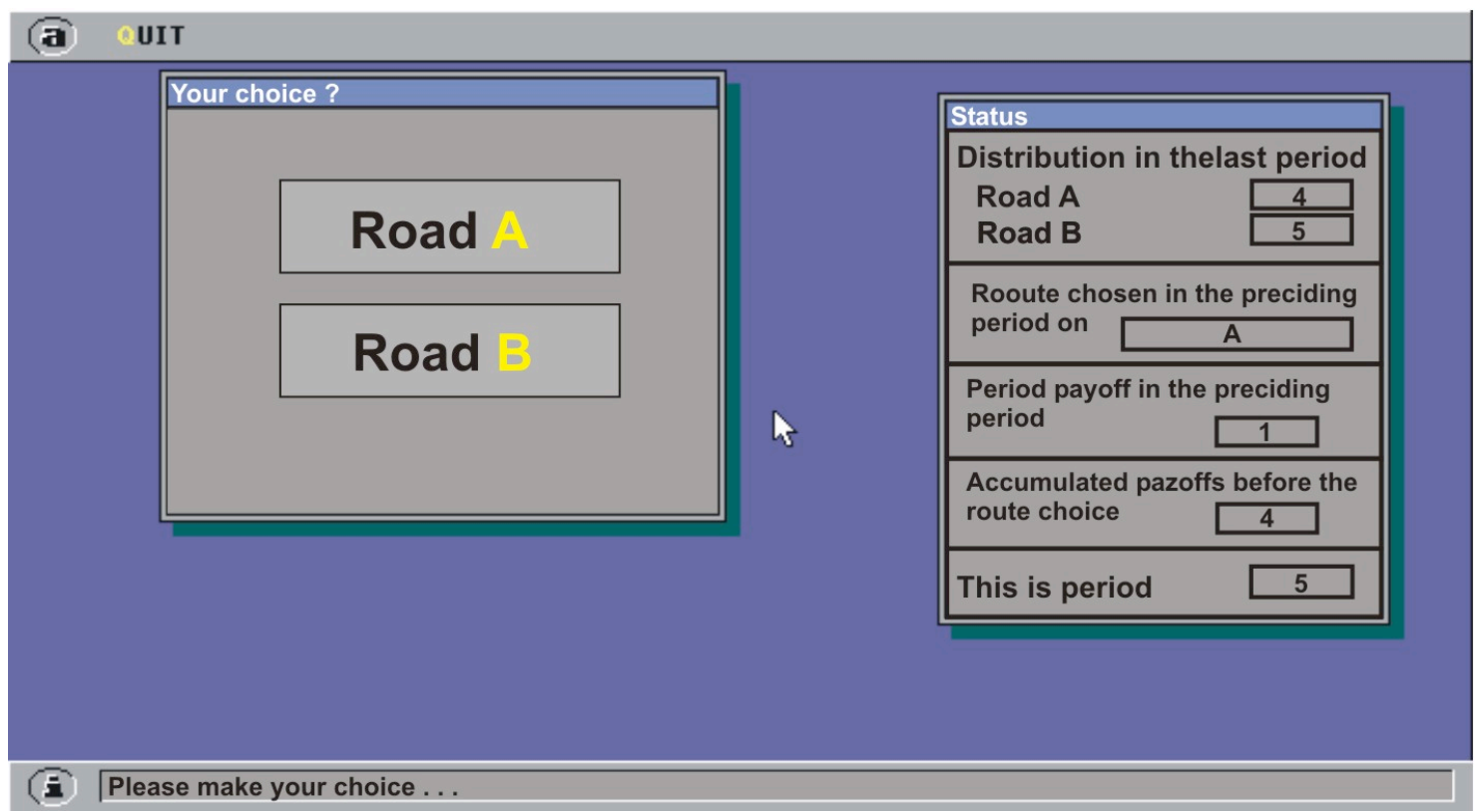

